ARTIGO ORIGINAL

\title{
Influência da incorporação de lignina Kraft à resina ureia-formaldeído nas propriedades tecnológicas de painéis aglomerados convencionais
}

\author{
Influence of the incorporation of Kraft lignin to the urea-formaldehyde \\ adhesive on the technological properties of conventional chipboards
}

\author{
Sandra Stramosk Luckman ${ }^{1}$ (D), Alexsandro Bayestorff da Cunha ${ }^{1}$ (D), Polliana D'Angelo Rios ${ }^{1}$ (D), Paula \\ Zanatta $^{1}$ (D) \\ ${ }^{1}$ Universidade do Estado de Santa Catarina - UDESC, Lages, SC, Brasil
}

Como citar: Luckman, S. S., Cunha, A. B., Rios, P. D., \& Zanatta, P. (2021). Influência da incorporação de Lignina Kraft à resina ureia-formaldeído nas propriedades tecnológicas de painéis aglomerados convencionais. Scientia Forestalis, 49(131), e3527. https://doi.org/10.18671/scifor.v49n131.10

\begin{abstract}
Resumo
O objetivo do estudo foi avaliar o efeito da adição de lignina Kraft à resina ureia-formaldeído comercial (UF) nas propriedades tecnológicas de painéis aglomerados. A matéria-prima utilizada foi composta por partículas de Pinus taeda L., UF, emulsão de parafina e dois tipos de lignina, os quais se diferenciavam pelo processo de extração. As ligninas foram analisadas de forma qualitativa, semi-quantitativa e por espectroscopia de infravermelho por transformada de Fourier. O delineamento experimental utilizado na avaliação dos painéis foi inteiramente casualizado com arranjo bifatorial, sendo as ligninas I e II os fatores e os diferentes percentuais aplicados à UF $(10,20$ e $30 \%)$ os níveis. Os painéis foram produzidos com densidade nominal de $0,700 \mathrm{~g} . \mathrm{cm}^{-3}, 12 \%$ de adesivo (UF + lignina), 1,5\% de emulsão de parafina e ciclo de prensagem com $40 \mathrm{kgf}^{-\mathrm{cm}^{-2}}$, $180^{\circ} \mathrm{C}$ por 10 minutos. Os adesivos foram caracterizados pelo $\mathrm{pH}$, viscosidade, gel time, teor de cinzas e de sólidos; enquanto os painéis, pelas propriedades tecnológicas. Os resultados demonstraram que, apesar das diferenças em suas propriedades físicas e nos processos de extração, ambas as ligninas apresentaram composições químicas semelhantes e que a adição percentual gradativa das ligninas à UF prejudicou a estabilidade dimensional e as propriedades de resistência e rigidez dos painéis. Desta forma, conclui-se que a combinação simples entre a UF e as duas ligninas, juntamente com as variáveis de processo utilizadas não são recomendadas para se atingir os parâmetros mínimos exigidos pelas normas de qualidade.
\end{abstract}

Palavras-chave: Painéis reconstituídos; Lignina Kraft; Adesivo termofixo.

\begin{abstract}
\end{abstract}
The aim of this study was to evaluate the effect of adding Kraft lignin to commercial urea-formaldehyde resin (UF) on the technological properties of chipboard panels. The raw material used was composed of Pinus taeda L. particles, UF, paraffin emulsion and two types of lignin, which differed by the extraction process. Lignins were analyzed qualitatively, semi-quantitatively and by Fourier transform infrared spectroscopy. The experimental design used in the evaluation of the panels was completely randomized with bi-factorial arrangement, being the lignins I and II the factors and the different percentages applied to the UF $(10,20$ and $30 \%$ ) the levels. The panels were produced with a nominal density of $0.70 \mathrm{~g} \cdot \mathrm{cm}^{-3}, 12 \%$ adhesive (UF + lignin), $1.5 \%$ paraffin emulsion and pressing cycle at $40 \mathrm{kgf.cm}^{-2}, 180^{\circ} \mathrm{C}$ for 10 minutes. The adhesives were characterized by $\mathrm{pH}$, viscosity, gel time, ash and solids content; while the panels, for the technological

Fonte de financiamento: Nenhuma.

Conflito de interesse: Nada a declarar.

Autor correspondente: alexsandro.cunha@udesc.br

Recebido: 4 dezembro 2019.

Aceito: 16 dezembro 2020.

Editor: Paulo Henrique Müller Silva.

(c) (i) Este é um artigo publicado em acesso aberto (Open Access) sob a licença Creative Commons Attribution, que permite uso, distribuição e reprodução em qualquer meio, sem restrições desde que o trabalho original seja corretamente citado. 
properties. The results showed that, despite the differences in their physical properties and extraction processes, both lignins presented similar chemical compositions and that the gradual percentage addition of lignins to the UF impaired the dimensional stability and the strength and stiffness properties of the panels. Thus, it is concluded that the simple combination between the UF and the two lignins, together with the process variables used are not recommended to achieve the minimum parameters required by the quality standards.

Keywords: Reconstituted panels; Kraft lignin; Thermoset adhesive.

\section{INTRODUÇÃO}

A indústria de papel e celulose possui grande representatividade para a economia do Brasil e para o setor de base florestal, devido à geração de receitas, aos grandes investimentos, à geração de empregos diretos e indiretos e ao impacto sobre outros setores econômicos interligados à sua cadeia produtiva.

Dentre os processos químicos de polpação, o Kraft é o mais utilizado pelas indústrias de celulose e papel, representando $90 \%$ de toda a produção mundial (Chávez-Sifontes \& Domine, 2013). Neste processo, a madeira é transformada em cavacos, que são colocados em contato com solução alcalina forte em vasos sob pressão e temperatura, com intuito de atacar e dissolver a lignina, formando ao final, a pasta celulósica.

A maioria das indústrias queima na caldeira de recuperação todo o licor preto forte produzido no cozimento, o qual é um líquido rico em substâncias orgânicas inorgânicas e extrativos. Esse licor possui constituintes da madeira na forma solubilizada, tal como a lignina.

A lignina é uma macromolécula com estrutura aromática polimérica, tridimensionalmente complexa, constituída de um polímero, formado por ligações cruzadas. Possui anéis benzênicos interligados por átomos de carbono, baixa solubilidade em água e alto conteúdo fenólico (Spiridon, 2018). Dessa forma, a lignina Kraft, tem potencial para torna-se um componente de grande importância para a indústria química (Collares, 2015), por meio da separação e comercialização dos subprodutos, que são atualmente direcionados à queima.

Devido às suas características, os subprodutos da lignina são usados, entre outras aplicações, como agentes dispersantes que dão fluidez e estabilização ao concreto, corantes têxteis, pesticidas, baterias e produtos de cerâmica, ou ainda como aditivos em alimentos para animais e briquetes (Nali et al., 2016). Outro setor que a lignina está despertando interesse é o de adesivos, em especial, o utilizado na indústria de painéis reconstituídos, onde, pode ser utilizado como aditivo aos adesivos comerciais, a fim de aumentar a qualidade dos painéis, de diminuir a toxicidade de seus processos e de reduzir os custos de produção.

A maioria dos estudos com adição de lignina ao adesivo para produção de painéis, está concentrada na modificação do adesivo fenol-formaldeído. Outros, como Freitas \& Lenz (2019), Carvalho et al. (2014a) e Rosa \& Hillig (2014), trabalharam com adesivo uréia-formaldeído modificada com tanino e lignossulfonato de amônio.

Considerando a oferta de lignina e a possibilidade de sua obtenção a partir de processos de recuperação do licor preto, assim como, o notável mercado de painéis reconstituídos de madeira, o presente estudo teve por objetivo avaliar a influência da incorporação de lignina Kraft à resina ureia-formaldeído comercial nas propriedades tecnológicas de painéis aglomerados convencionais.

\section{MATERIAL E MÉTODOS}

A matéria-prima utilizada foi composta por partículas sliver de Pinus taeda L. com $4 \pm 1 \%$ de umidade, fornecidas por uma indústria de painéis particulados de Santa Catarina; resina ureiaformaldeído comercial (UF); emulsão de parafina; e dois tipos de lignina Kraft em pó, que se diferenciavam em função do processo de extração a partir do licor preto Kraft, o qual era 
proveniente do cozimento de madeiras de Eucalyptus spp. em processo adaptado do LignoBoost. Sendo denominadas no presente estudo de Lignina I (LI) e Lignina II (LII).

A identificação qualitativa e semi-quantitativa dos componentes das ligninas foi realizada por análise de espectroscopia de infravermelho por transformada de Fourier (FTIR) no FT/IR - 4100, com range de $4000-600 \mathrm{~cm}^{-1}$, resolução de $4 \mathrm{~cm}^{-1}$ em módulo ATR. Foram realizadas análises físico-químicas das ligninas para teor de cinzas (Technical Association of the Pulp and Paper Industry, 1998), teor de sólidos (American Society for Testing and Materials, 2013) e pH (Associação Brasileira de Normas Técnicas, 1999).

O modelo experimental utilizado para a avaliação das propriedades dos painéis foi o delineamento inteiramente casualizado com arranjo bifatorial, tendo como fatores as duas ligninas (I e II) em três níveis de adição à UF (10, 20 e 30\%), totalizando 18 painéis, divididos em 6 tratamentos com 3 repetições cada, mais 3 painéis utilizados como testemunha, sem adição de lignina à UF.

Foram realizadas análises laboratoriais de pH (Associação Brasileira de Normas Técnicas, 1999), viscosidade Brookfield (American Society for Testing and Materials, 1998), gel time (American Society for Testing and Materials, 1999), teor de cinzas (Technical Association of the Pulp and Paper Industry, 1998) e teor de sólidos (American Society for Testing and Materials, 2013), tanto na UF, quanto nos adesivos formados com adição de lignina.

Os painéis homogêneos foram produzidos com densidade nominal de $0,700 \mathrm{~g} \cdot \mathrm{cm}^{-3}$, dimensões de $40 \times 40 \times 1,5 \mathrm{~cm}, 12 \%$ de adesivo (UF + lignina), 1,5\% de emulsão de parafina sobre a massa seca das partículas, sem catalisador para aumentar o tempo em aberto, prensagem a frio com $5 \mathrm{kgf} . \mathrm{cm}^{-2}$ por 5 minutos, e ciclo de prensagem final para consolidação do painel com $40 \mathrm{kgf} . \mathrm{cm}^{-2}$ de pressão, $180^{\circ} \mathrm{C}$ de temperatura por 10 minutos, adicionandose mais 1,5 minuto para fechamento dos pratos da prensa e outro 1,5 minuto para abertura.

A incorporação da lignina a resina UF foi realizada de forma simples e direta, por meio de um mix proporcional em um béquer com movimentação contínua por meio de um agitador magnético, por um tempo de 15 minutos. Posteriormente, o mix foi aplicado as partículas em um tambor rotativo, dotado de uma pistola pulverizadora acionada por um compressor. A massa de partículas e adesivo, formou o colchão, que foi pré-prensado a frio e prensado a quente para proporcionar a cura do adesivo e a consolidação do painel.

A determinação das propriedades físicas de densidade, absorção de água e inchamento em espessura, juntamente com a tração perpendicular foi realizada de acordo com a ASTM D1037 (American Society for Testing and Materials, 1993); enquanto a flexão estática, pela DIN 52362 (Deutsches Institut für Normung, 1982) em função do menor comprimento do corpo de prova, e o arrancamento de parafuso pela NBR 14810 (Associação Brasileira de Normas Técnicas, 2006) devido a ASTM não descrever o ensaio.

A razão de compactação (RC) foi obtida pela relação entre a densidade do painel e da madeira $\left(0,380 \mathrm{~g} \cdot \mathrm{cm}^{-3}\right)$. O perfil de densidade foi determinado em um densitômetro de raiosX IMAL, modelo DPX300, versão 2.7.4.9-1.

A análise das ligninas e dos adesivos foi realizada de forma descritiva, enquanto a análise do FTIR considerou a absorbância de cada composto presente nas ligninas em diferentes bandas de absorção.

Os resultados encontrados nos ensaios físicos e mecânicos dos painéis foram testados quanto à presença de outliers (boxplot), à normalidade de distribuição dos dados (ShapiroWilk) e à homogeneidade de variâncias (Levene). Por fim, foi aplicada a Análise da Variância, e quando necessário o teste de Scott-Knott a 95\% de probabilidade de acerto. Para a comparação dos tratamentos com a testemunha, foi aplicado o Teste de Dunnet. Os valores médios obtidos nos ensaios, foram comparados com os parâmetros de referência da NBR 14810 (Associação Brasileira de Normas Técnicas, 2013), da EN 312-2 (Comité Européen de Normalisation, 2003), da CSA 0437 (Canadian Standards Association, 1993) e da ANSI A208.1 (American National Standards Institute, 2009). 


\section{RESULTADOS E DISCUSSÃO}

Nos valores relativos às características físicas e químicas das ligninas (Tabela 1), é possível observar grande diferença de $\mathrm{pH}$, decorrente, provavelmente, do processo de extração das ligninas do licor preto Kraft, onde a $\mathrm{LI}$ foi lavada mais vezes com $\mathrm{H}_{2} \mathrm{SO}_{4}$ e $\mathrm{H}_{2} \mathrm{O}$, enquanto a LII foi somente submetida à reação com $\mathrm{CO}_{2}$. Os valores extremos de $\mathrm{pH}$ observados, caracterizam as ligninas como muito ácida (I) e muito alcalina (II), trazendo consequências negativas ao processo de colagem, uma vez que a colagem com adesivo ácido pode ocasionar baixo tempo em aberto e uma provável pré-cura, não proporcionando a fluidez, a molhabilidade e a absorção do adesivo pelas partículas, prejudicando a coesão e a resistência. Já para o adesivo alcalino, é o inverso, pois necessita de maior tempo para atingir a cura, refletindo em perda de produção ou necessidade da adição de catalisador.

Tabela 1. Valores médios das análises físico-químicas das ligninas.

\begin{tabular}{ccc}
\hline Análise & Lignina I & Lignina II \\
\hline $\mathrm{Ph}$ & 3,70 & 9,30 \\
Teor de cinzas (\%) & 1,02 & 26,14 \\
Teor de sólidos (\%) & 95,57 & 97,11 \\
\hline
\end{tabular}

Como o teor de cinzas (Tabela 1), em sua maioria, é devido aos sais inorgânicos remanescentes do cozimento (Giummarella et al., 2016), também pode-se atribuir o maior valor da LII a questões do processo de extração. Zhou (2014) cita que é necessário um baixo valor de cinzas, com limite superior de 3\%, de modo a favorecer a reatividade da lignina. Já para ElMansouri (2006), o ideal é que a lignina possua no máximo 0,5\%, também para garantir maior pureza e reatividade com o formaldeído presente na resina. Dessa forma, a LI tende a ser mais reativa, visto que apresenta menores ligações com os compostos inorgânicos do cozimento podendo proporcionar melhor interações químicas com a UF.

Observa-se na Figura 1, o FTIR das ligninas, onde fica evidenciado que os principais picos de absorção no infravermelho são característicos da macromolécula, e que nenhuma das duas sofreu alterações em sua composição química, apesar de terem sido obtidas por diferentes processos.

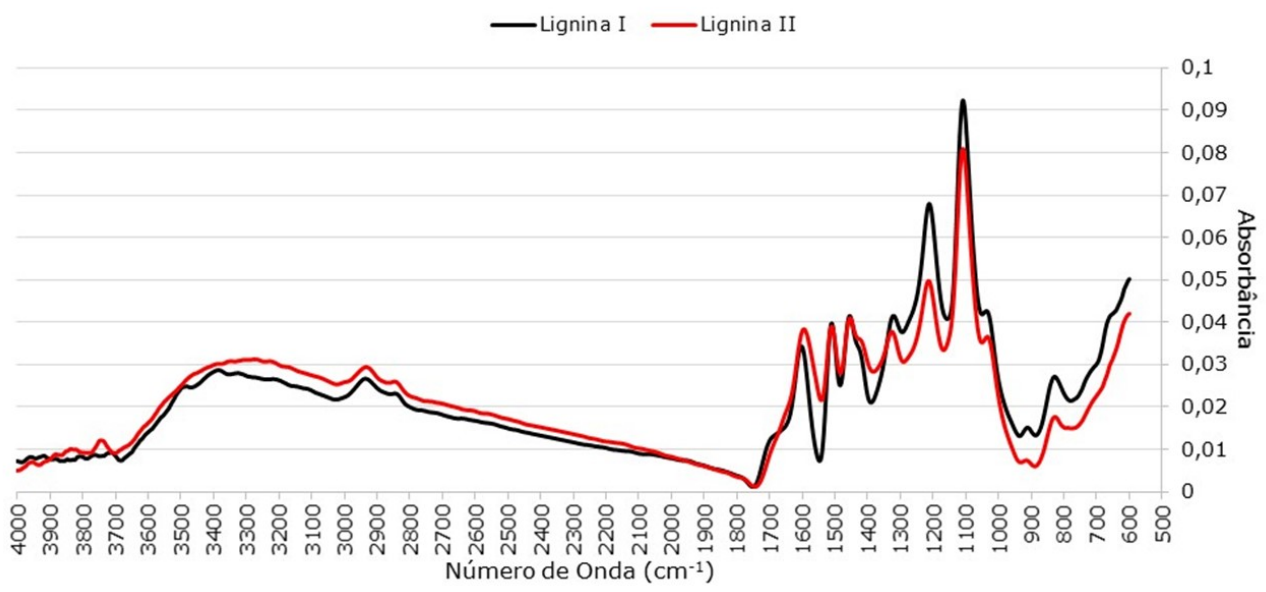

Figura 1. Resultados de FTIR para as duas ligninas. 
Comumente, os espectros de lignina mostram uma banda ampla entre 3400 e $3600 \mathrm{~cm}^{-1}$, atribuída aos grupos hidroxílicos de ácidos fenólicos e carboxílicos, os quais estão associados aos compostos fenólicos e estruturas alifáticas da lignina (You \& Xu, 2016). A banda de absorção a $1703 \mathrm{~cm}^{-1}$ é atribuída às porções de carbonila dos grupos aldeído e cetona. Segundo Negrão et al. (2015), a falta da banda espectral nessa região sugere modificação química da maioria dos grupos carbonila sob condições de alto $\mathrm{pH}$, justificando a ausência dessas vibrações na LII. As bandas em 1300 e $1200 \mathrm{~cm}^{-1}$ indicam, respectivamente, a presença de siringil e guaiacil em ambas, contudo em maior intensidade desta última em LI, sugerindo maior reatividade da LI que a LII, uma vez que a lignina siringil apresenta um grupo metil a mais que a do tipo guaiacil (Magalhães et al., 2019). Novamente, o baixo $\mathrm{pH}$ da $\mathrm{LI}$ demonstra que esta passou por mais processos ou maior tempo de lavagem com $\mathrm{H}_{2} \mathrm{SO}_{4}$, visto que a banda em $1100 \mathrm{~cm}^{-1}$ é responsável pela deformação no plano $\mathrm{C}-\mathrm{H}$ aromático interligado às unidades siringil, o que não distinguiu expressivamente devido à diferença de pH entre as ligninas. Já a banda de absorção em $1414 \mathrm{~cm}^{-1}$ apresentou vibração na LII e refere-se à ligação C-C do anel aromático com a lignina, reforçando novamente que o procedimento para extração desta foi menos agressivo, ocorrendo menor quebra das ligações. Dessa forma, os diferentes processos de extração de ambas as ligninas não ocasionaram grandes diferenças estruturais entre elas, demonstrando que o maior pH da LII ocasionou menor quebra de ligações em sua estrutura e reforçando que a LI se apresentou mais reativa devido à maior presença de unidades guaiacil.

A Tabela 2 apresenta a influência da adição das ligninas à UF, demonstrando que houve alterações em todas as características; enquanto no pH ocorreu diminuição para a $\mathrm{LI}$ e aumento para a LII; na viscosidade e no teor de sólidos, o aumento foi para ambas. Já para gel time, houve redução de forma mais acentuada com adição da LI, visto que com o aumento do percentual de LI (ácida) na UF observa-se severa redução do pH, ocasionando diminuição no tempo de cura, sendo corroborada pela redução dos valores de gel time, que chegou a 47 segundos. Ainda em relação à LI, observou-se aumento acentuado da viscosidade e do teor de sólidos, dificultando a pulverização do adesivo sobre as partículas devido ao entupimento dos bicos aspersores e ainda, sua penetração na estrutura da madeira, podendo gerar linha de cola não ancorada.

Tabela 2. Propriedades físicas e químicas dos adesivos (UF + ligninas).

\begin{tabular}{|c|c|c|c|c|c|c|c|}
\hline \multirow{2}{*}{ Análise } & \multirow{2}{*}{ UF } & \multicolumn{3}{|c|}{ UF + Lignina I } & \multicolumn{3}{|c|}{ UF + Lignina II } \\
\hline & & $10 \%$ & $20 \%$ & $30 \%$ & $10 \%$ & $20 \%$ & $30 \%$ \\
\hline $\mathrm{Ph}$ & 7,96 & 5,81 & 5,20 & 4,11 & 7,96 & 8,09 & 8,20 \\
\hline Viscosidade (cP) & 375 & 670 & 1425 & 1730 & 1272 & 1765 & 2715 \\
\hline Gel time (s) & 371 & 78 & 48 & 47 & 292 & 278 & 151 \\
\hline Teor de sólidos (\%) & 64,90 & 74,02 & 76,41 & 78,81 & 74,17 & 76,72 & 79,27 \\
\hline
\end{tabular}

O acréscimo da quantidade de LII à UF também resultou no aumento do teor de sólidos, ocasionando aumento expressivo da viscosidade, que com $30 \%$, chegou a 7,24 vezes maior que a UF pura. Akpabio (2012) analisou as propriedades do adesivo à base de UF em diferentes faixas de $\mathrm{pH}$, e verificou que a viscosidade aumenta tanto para $\mathrm{pH}$ ácido, quanto para alcalino, apresentando maior aumento em $\mathrm{pH}$ ácido. Assim como, o gel time sofre decréscimo até $\mathrm{pH}$ 4,5 e aumenta em pH 2,0 a 3,0. Em contrapartida, o tempo de gelatinização decai em pH acima de 7,0. Já Silva et al. (2019) ao realizarem modificação da UF com adição de lignossulfonato do processo sulfito em pó diluído em água, obtiveram redução da viscosidade, do pH e do teor de sólidos do adesivo, não ocorrendo formação da 
fase gel, mesmo utilizando catalisador. Dessa forma, percebe-se que a UF não reage facilmente com substituintes do formaldeído, havendo necessidade de proporcionar condições que melhorem sua reatividade, tal como incluir a lignina durante a síntese da resina em que ureia e formaldeído serão reticulados, ou ainda, modificá-la com hidroximetilação ou glioxilação.

$\mathrm{Na}$ Tabela 3, pode ser observado que houve diferença estatística nos valores de densidade e razão de compactação $(R C)$ em todos os tratamentos entre as quantidades incorporadas, tanto para LI, quanto para LII. Observa-se também que os tratamentos T4 e T7 (30\% de lignina) apresentaram os menores valores e não foram equivalentes aos valores da testemunha. Constata-se também, que não houve diferença significativa entre as duas ligninas, independentemente da quantidade aplicada.

Tabela 3. Valores médios de densidade e razão de compactação (RC).

\begin{tabular}{|c|c|c|c|c|}
\hline \multirow{2}{*}{$\begin{array}{c}\text { Composição do } \\
\text { painel }\end{array}$} & \multicolumn{2}{|c|}{ Densidade $\left(\mathrm{g} \cdot \mathrm{cm}^{-3}\right)$} & \multicolumn{2}{|c|}{ Razão de compactação } \\
\hline & Lignina I & Lignina II & Lignina I & Lignina II \\
\hline 90UF:10L & ${ }^{\mathrm{T} 2} 0,630 \mathrm{Aa}$ & ${ }^{\mathrm{T} 5} 0,620 \mathrm{Aa}$ & $\mathrm{T} 21,65 \mathrm{Aa}$ & T51,64 Aa \\
\hline 80UF:20L & T3 $0,620 \mathrm{Aa}$ & ${ }^{\mathrm{T}} 0,620 \mathrm{Aa}$ & T31,63 Aa & ${ }^{\mathrm{T}}{ }^{1,64 \mathrm{Aa}}$ \\
\hline 70UF:30L & $\mathrm{T}^{\mathrm{T}} 0,600 \mathrm{Ba} *$ & ${ }^{\top 7} 0,600 \mathrm{Ba}^{*}$ & ${ }^{\top 4} 1,58 \mathrm{Ba}^{*}$ & $\mathrm{~T} 71,54 \mathrm{Ba} *$ \\
\hline $100 \mathrm{UF}$ & \multicolumn{2}{|c|}{ Testemunha 0,65} & \multicolumn{2}{|c|}{ Testemunha1,72 } \\
\hline Densidade nor & \multicolumn{2}{|c|}{0,700} & & \\
\hline $\begin{array}{l}\text { NBR } 14810 \text { (Asso } \\
\text { Brasileira de No } \\
\text { Técnicas, 20 }\end{array}$ & \multicolumn{2}{|c|}{0,550 a 0,750} & & \\
\hline $\begin{array}{r}\text { EN 312-2 (Con } \\
\text { Européen o } \\
\text { Normalisation, }\end{array}$ & \multicolumn{2}{|c|}{0,550 a 0,750} & & \\
\hline $\begin{array}{l}\text { ANSI A208.1 (Am } \\
\text { National Stand } \\
\text { Institute, } 200\end{array}$ & \multicolumn{2}{|c|}{0,604 a 0,800} & & \\
\hline \multicolumn{3}{|c|}{ Moslemi (1974); Maloney (1993) } & \multicolumn{2}{|c|}{$>1,3$} \\
\hline
\end{tabular}

Médias seguidas de mesma letra maiúscula na coluna e mesma letra minúscula na linha não apresentam diferença significativa entre si pelo Teste de Scott-Knott; *: diferença estatística em relação ao tratamento testemunha pelo teste de Dunnett.

As diferenças estatísticas observadas e a não obtenção da densidade nominal podem ser atribuídas à especificidade das condições laboratoriais, tendo em vista que a distribuição do colchão é manual e que as quantidades de partículas e de adesivo são previamente calculadas em função da densidade nominal e das dimensões desejadas. Esta situação é recorrente, sendo observada em diversos trabalhos como Andrade et al. (2018), que encontraram densidade dos painéis variando entre 0,620 a 0,650 g. $\mathrm{cm}^{-3}$; Martins et al. (2014) que obtiveram densidade entre 0,640 e $0,670 \mathrm{~g} \cdot \mathrm{cm}^{-3}$ e nominal de $0,700 \mathrm{~g} \cdot \mathrm{cm}^{-3} \mathrm{em}$ ambos os trabalhos; e Trianoski et al. (2013) com densidade de 0,680 g.cm $\mathrm{cm}^{-3}$ e nominal de $0,750 \mathrm{~g} \cdot \mathrm{cm}^{-3}$.

Tomando-se como referência o estabelecido pelas normas NBR 14810 (Associação Brasileira de Normas Técnicas, 2013) e EN 312-2 (Comité Européen de Normalisation, 2003), constata-se que os painéis de todos os tratamentos podem ser classificados como de média densidade, entretanto, nenhum deles atingiu o limite da ANSI A208.1 (American National Standards Institute, 2009). Já para RC, 
todos os tratamentos obtiveram valores superiores ao mínimo estabelecido por Moslemi (1974) e Maloney (1993). Segundo Andrade et al. (2018), essa propriedade é um parâmetro de grande importância na resistência mecânica de painéis, uma vez que painéis com maior RC, tendem a apresentar propriedades mecânicas superiores e maiores coeficientes de absorção.

Os valores de perfil de densidade dos painéis são apresentados na Tabela 4, na qual observa-se que houve diferença estatística somente na quantidade aplicada para a LII (30\%), ou seja, ambas as ligninas apresentaram comportamento equivalente em relação à distribuição da densidade ao longo da espessura.

Tabela 4. Valores médios do perfil de densidade dos painéis.

\begin{tabular}{|c|c|c|c|c|c|c|}
\hline \multirow{3}{*}{$\begin{array}{c}\text { Composição } \\
\text { do painel }\end{array}$} & \multicolumn{6}{|c|}{ Perfil de densidade $\left(\mathrm{g} . \mathrm{cm}^{-3}\right)$} \\
\hline & \multicolumn{2}{|c|}{ Superfície Inferior } & \multicolumn{2}{|c|}{ Miolo } & \multicolumn{2}{|c|}{ Superfície Superior } \\
\hline & Lignina I & Lignina II & Lignina I & Lignina II & Lignina I & Lignina II \\
\hline 90UF:10L & ${ }^{\mathrm{T}} 20,816 \mathrm{Aa}$ & ${ }^{\mathrm{T}} 0,797 \mathrm{Aa}$ & ${ }^{\mathrm{T}} 0,561 \mathrm{Aa}$ & ${ }^{\mathrm{T}} 0,557 \mathrm{Aa}$ & ${ }^{\mathrm{T}} 0,746 \mathrm{Aa}$ & ${ }^{\mathrm{T}} 0,764 \mathrm{Aa}$ \\
\hline 80UF:20L & ${ }^{\mathrm{T} 3} 0,775 \mathrm{Aa}$ & ${ }^{\mathrm{T}} 0,718 \mathrm{Aa}$ & ${ }^{\mathrm{T} 3} 0,567 \mathrm{Aa}$ & ${ }^{\mathrm{T}} 0,524 \mathrm{Aa}$ & ${ }^{\mathrm{T} 3} 0,767 \mathrm{Aa}$ & ${ }^{\mathrm{T}} 0,734 \mathrm{Aa}$ \\
\hline 70UF:30L & ${ }^{\mathrm{T}} 0,766 \mathrm{Aa}$ & ${ }^{\mathrm{T}} 0,595 \mathrm{Ba} *$ & ${ }^{\mathrm{T}} 0,532 \mathrm{Aa}$ & ${ }^{77} 0,439 \mathrm{Ba*}$ & ${ }^{\mathrm{T}} 0,733 \mathrm{Aa}$ & ${ }^{\top 7} 0,569 \mathrm{Ba}^{*}$ \\
\hline 100UF & \multicolumn{2}{|c|}{ Testemunha 0,825} & \multicolumn{2}{|c|}{ Testemunha 0,569} & \multicolumn{2}{|c|}{ Testemunha 0,827} \\
\hline
\end{tabular}

Médias seguidas de mesma letra maiúscula na coluna e mesma letra minúscula na linha não apresentam diferença significativa entre si pelo Teste de Scott-Knott; *: diferença estatística em relação ao tratamento testemunha pelo teste de Dunnett.

Verifica-se maior densidade nas camadas da superfície e menor na parte central dos painéis, formando um perfil em formato de " $M$ ", que é característico deste painel (Gamage \& Setunge, 2015). A variação de densidade entre as camadas ocorre devido ao tempo de fechamento da prensa e a diferenças na transferência de temperatura das camadas externas para a camada interna do painel durante o estágio de compressão das partículas até atingir a espessura final do colchão (Moslemi, 1974). Durante a prensagem dos painéis, as partículas das camadas externas recebem calor antes das partículas da camada central e plasticizam, oferendo menor resistência à compressão, de modo que a camada externa fica mais densificada. Quando a temperatura chega à camada central e os pratos da prensa encostam nos separadores, não é possível continuar comprimindo as partículas da camada central. Fica evidente que a presença da lignina nos adesivos potencializou essa plasticização, devido à temperatura de transição vítrea da lignina, explicando o acréscimo da densidade da camada externa. Algumas propriedades, como a resistência à flexão, são significativamente aumentadas pela presença de gradiente de densidade, enquanto outras, como resistência à tração perpendicular, são afetadas negativamente por esta distribuição de densidade no sentido da espessura do painel (Gamage \& Setunge, 2015; Kelly, 1977).

A estabilidade dimensional dos painéis é reflexo da quantidade de água absorvida durante o período de imersão e do inchamento percentual em espessura. Na Tabela 5 observa-se equivalência entre os valores médios encontrados para as duas ligninas, no entanto, o aumento do percentual de lignina no adesivo gerou aumento nos coeficientes, principalmente para LII a 30\% (T7). Observa-se também, que todos os tratamentos apresentaram diferença estatística em relação ao tratamento testemunha tanto para absorção, com superioridade entre $36 \%$ e $89 \%$, quanto para o inchamento, com intervalo entre $54 \%$ e $137 \%$, ambos para LI (10\%) e LII (30\%). 
Tabela 5. Valores médios para as variáveis relacionadas à estabilidade dimensional.

\begin{tabular}{|c|c|c|c|c|}
\hline \multirow{2}{*}{$\begin{array}{c}\text { Composição do } \\
\text { painel }\end{array}$} & \multicolumn{2}{|c|}{ Absorção de água (\%) } & \multicolumn{2}{|c|}{ Inchamento em espessura (\%) } \\
\hline & Lignina I & Lignina II & Lignina I & Lignina II \\
\hline 90UF:10L & T2 106,30Aa* & T5 99,61Aa* & T231,78Aa* & T535,37Aa* \\
\hline 80UF:20L & T3112,28Aa* & T6102,03Aa* & T336,68Aa* & T636,13Aa* \\
\hline 70UF:30L & T4120,49Aa* & Т7148,19Ba* & T439,71Aa* & ${ }^{\top 7} 49,00 \mathrm{Ba} *$ \\
\hline $100 U F$ & \multicolumn{2}{|c|}{ Testemunha 78,24 } & \multicolumn{2}{|c|}{ Testemunha 20,65} \\
\hline $\begin{array}{c}\text { CSA } 0437 \text { (Canadian } \\
\text { Standards Association, } \\
\text { 1993) }\end{array}$ & \multicolumn{2}{|c|}{$<15 \%$} & & \\
\hline $\begin{array}{c}\text { NBR } 14810 \\
\text { (Associação Brasileira } \\
\text { de Normas Técnicas, } \\
\text { 2013) }\end{array}$ & & & \multicolumn{2}{|c|}{$<18 \%$} \\
\hline $\begin{array}{l}\text { ANSI A208.1 (American } \\
\text { National Standards } \\
\text { Institute, 2009) }\end{array}$ & & & \multicolumn{2}{|c|}{$<40 \%$} \\
\hline $\begin{array}{l}\text { EN 312-2 (Comité } \\
\text { Européen de } \\
\text { Normalisation, 2003) }\end{array}$ & & & \multicolumn{2}{|c|}{$<15 \%$} \\
\hline
\end{tabular}

Médias seguidas de mesma letra maiúscula na coluna e mesma letra minúscula na linha não apresentam diferença significativa entre si pelo Teste de Scott-Knott; *: diferença estatística em relação ao tratamento testemunha pelo teste de Dunnett.

Elevados valores médios também foram encontrados por Ferreira (2017), que utilizou adesivo lignina-ureia-formaldeído formulado em laboratório para a produção de painéis MDP de Eucalyptus spp. com densidade de $0,590 \mathrm{~g} . \mathrm{cm}^{-3}$. Para absorção de água (AA), a autora encontrou $65 \%$ após 2 horas de imersão e $97 \%$ para 24 horas, e para inchamento em espessura (IE), $24 \%$ para 2 horas e $32 \%$ para 24 horas. Todos os tratamentos excederam o limite máximo da CSA 0437 (Canadian Standards Association, 1993) para AA 24h, inclusive a testemunha, resultado observado na maioria dos trabalhos com painel reconstituído de madeira. Já para o IE 24h, apenas o tratamento com adição de $30 \%$ de LII não atendeu aos requisitos da norma ANSI A208.1 (American National Standards Institute, 2009). Elevados valores para estabilidade dimensional podem ser atribuídos à alta RC dos painéis devido à maior quantidade de material por unidade de volume; à baixa reatividade da lignina, que apesar de ser semelhante ao fenol, apresenta grupos metil ligados ao anel aromático, dificultando ou até impossibilitando a ligação química com outros compostos; à ineficiência do processo de adesão (substrato / partículas + adesivo) em função de variáveis como pH, viscosidade, gel time e teor de sólidos; ao alto teor de cinzas da LII que pode prejudicar a reatividade com o formaldeído presente na resina; e à diferença no perfil de densidade dos painéis, visto que menores variações no perfil proporcionam menor liberação da tensão de compressão quando expostos à água.

A influência do tipo e da quantidade de lignina adicionada à UF sobre o módulo de elasticidade (MOE) e o módulo de ruptura (MOR) está apresentada na Tabela 6, na qual observase equivalência dos valores médios de ambas as ligninas para MOE e superioridade da LI para MOR. Em relação à quantidade aplicada, houve redução significativa nos valores de MOE à medida que aumentou-se o percentual de ligninas adicionado à UF. 
Tabela 6. Valores médios para módulo de ruptura e elasticidade à flexão estática.

\begin{tabular}{|c|c|c|c|c|}
\hline \multirow{3}{*}{$\begin{array}{l}\text { Composição do } \\
\text { painel }\end{array}$} & \multicolumn{4}{|c|}{ Flexão Estática } \\
\hline & \multicolumn{2}{|c|}{ MOE (MPa) } & \multicolumn{2}{|c|}{ MOR (MPa) } \\
\hline & Lignina I & Lignina II & Lignina II & Lignina II \\
\hline 90UF:10L & T21085,23Aa* & ${ }^{T 5} 922,46 A a^{*}$ & ${ }^{\mathrm{T}} 11,77 \mathrm{Aa}$ * & ${ }^{\mathrm{T}} 8,09 \mathrm{Ab} *$ \\
\hline $80 U F: 20 \mathrm{~L}$ & ${ }^{\mathrm{T}} 3817,21 \mathrm{Ba}^{*}$ & T6807,53Ba* & T310,44Aa* & ${ }^{16} 6,66 \mathrm{Ab} *$ \\
\hline 70UF:30L & Т4 $761,64 \mathrm{Ba}^{*}$ & $\mathrm{~T} 7549,72 \mathrm{Ca} *$ & ${ }^{T} 410,23 \mathrm{Aa}$ * & ${ }^{77} 5,06 \mathrm{Bb}$ * \\
\hline 100UF & \multicolumn{2}{|c|}{ Testemunha 1835,41} & \multicolumn{2}{|c|}{ Testemunha 19,00} \\
\hline \multicolumn{2}{|c|}{$\begin{array}{c}\text { NBR } 14810 \text { (Associação } \\
\text { Brasileira de Normas Técnicas, } \\
\text { 2013) }\end{array}$} & 600 & \multicolumn{2}{|c|}{$>11,00$} \\
\hline \multicolumn{2}{|c|}{$\begin{array}{c}\text { EN 312-2 (Comité Européen de } \\
\text { Normalisation, 2003) }\end{array}$} & 600 & \multicolumn{2}{|c|}{$>13,00$} \\
\hline \multicolumn{2}{|c|}{$\begin{array}{c}\text { ANSI A208.1 (American } \\
\text { National Standards Institute, } \\
\text { 2009) }\end{array}$} & 550 & \multicolumn{2}{|c|}{$>10,00$} \\
\hline
\end{tabular}

Médias seguidas de mesma letra maiúscula na coluna e mesma letra minúscula na linha não apresentam diferença significativa entre si pelo Teste de Scott-Knott; *: diferença estatística em relação ao tratamento testemunha pelo teste de Dunnett.

Os valores médios de todos os tratamentos foram inferiores ao testemunha e, comparando-se com as normas de referência, observa-se que, com exceção do testemunha, nenhum tratamento atendeu aos parâmetros mínimos para MOE; já para MOR, todos os tratamentos relacionados ao testemunha e à LI atenderam à ANSI A208.1 (American National Standards Institute, 2009). Além dos valores médios de MOE e MOR terem ficado abaixo do tratamento testemunha e, em grande parte, dos parâmetros de referência, observou-se grande redução em relação ao estudo de Ferreira (2017) que incorporou lignina Kraft $(\mathrm{pH} 3,7)$ à formulação do adesivo ureia-formaldeído para produção de painéis MDP. A autora encontrou $3410 \mathrm{MPa}$ para $\mathrm{MOE}$ e $88,7 \mathrm{MPa}$ para MOR, cabe salientar que foi adicionada água na formulação dos adesivos para contornar a alta viscosidade e consequentemente, a ineficiência da penetração do adesivo. Outros estudos utilizando UF modificada também encontraram baixos valores de MOE e MOR, como em Freitas \& Lenz (2019) e Carvalho et al. (2014a) que trabalharam com tanino de casca de acácia, encontrando MOE entre 270 e $367 \mathrm{MPa}$ e MOR entre 1,66 e 2,28 MPa; e MOE entre 984,09 a $1739,11 \mathrm{MPa}$ e MOR entre 5,32 a 10,81MPa, respectivamente. Segundo Wu (1999), a resistência à flexão estática é influenciada pelo grau de adesão, densidade, perfil de densidade e ainda, RC. Com relação à densidade dos painéis, à RC e ao perfil de densidade, apenas os tratamentos com adição de $30 \%$ de ligninas apresentaram diferença significativa dos demais. Novamente, a UF modificada influenciou negativamente nas propriedades dos painéis produzidos, visto que o baixo $\mathrm{pH}$ da $\mathrm{LI}$, alto teor de cinzas da LII e o alto teor de sólidos de ambas as ligninas ocasionaram prejuízo na cura do adesivo e no seu escoamento pelas partículas.

Os valores médios de tração perpendicular (TP) estão apresentados na Tabela 7, onde observa-se que houve equivalência entre os valores médios de ambas as ligninas, e que o aumento da quantidade de lignina acrescentada à UF ocasionou decréscimo da propriedade, tanto que a partir de $20 \%$ de adição, nenhum dos tratamentos atendeu aos requisitos das normas de referência. Nota-se também, que todos os tratamentos produzidos com adição de LII diferiram da testemunha. 
Tabela 7. Valores médios para tração perpendicular (ligação interna).

\begin{tabular}{|c|c|c|c|}
\hline \multirow{2}{*}{ Composição do painel } & \multicolumn{3}{|c|}{ Tração perpendicular (MPa) } \\
\hline & Lignina I & & Lignina II \\
\hline 90UF:10L & ${ }^{\mathrm{T} 2} 0,46 \mathrm{Aa}$ & & ${ }^{\mathrm{T}} 0,37 \mathrm{Aa}$ * \\
\hline 80UF:20L & ${ }^{\mathrm{T}} 0,34 \mathrm{Ba}$ & & ${ }^{\mathrm{T}} 0,29 \mathrm{Ba}$ * \\
\hline 70UF:30L & ${ }^{\mathrm{T} 4} 0,30 \mathrm{Ca}$ & & ${ }^{\top 7} 0,19 \mathrm{Ca} *$ \\
\hline 100UF & \multicolumn{3}{|c|}{ Testemunha 0,61} \\
\hline $\begin{array}{l}\text { NBR } 14810 \text { (Associação Brasileira } \\
\text { de Normas Técnicas, 2013) }\end{array}$ & \multicolumn{3}{|c|}{$>0,35$} \\
\hline $\begin{array}{c}\text { EN 312-2 (Comité Européen de } \\
\text { Normalisation, 2003) }\end{array}$ & \multicolumn{3}{|c|}{$>0,35$} \\
\hline $\begin{array}{c}\text { CSA } 0437 \text { (Canadian Standards } \\
\text { Association, 1993) }\end{array}$ & \multicolumn{3}{|c|}{$>0,48$} \\
\hline $\begin{array}{l}\text { ANSI A208.1 (American National } \\
\text { Standards Institute, 2009) }\end{array}$ & \multicolumn{3}{|c|}{$>0,36$} \\
\hline
\end{tabular}

Médias seguidas de mesma letra maiúscula na coluna e mesma letra minúscula na linha não apresentam diferença significativa entre si pelo Teste de Scott-Knott; *: diferença estatística em relação ao tratamento testemunha pelo teste de Dunnett.

A TP fornece uma medida da integridade do painel, definindo quão bem o material do núcleo está colado (Kim et al., 2003), assim, para promover a melhoria na adesão das partículas e aumentar a TP, Iwakiri et al. (2004); Eleotério et al. (2000) e Wong et al. (1999), destacam que é necessário aumentar a densidade do painel, teor de resina e tempo e temperatura de prensagem. Sabendo-se que os parâmetros de processo (tempo e temperatura de prensagem) foram os mesmos, bem como, a densidade média dos painéis não apresentou diferença significativa (com exceção dos tratamentos com adição de $30 \%$ de lignina), adicionado ao fato que a ligação interna está diretamente ligada à qualidade da colagem dos painéis, novamente percebe-se a importância do $\mathrm{pH}$ do adesivo, além da sua viscosidade e do teor de sólidos. Uma perfeita adesão depende do ajuste do tempo e da temperatura de prensagem de acordo com o pH do adesivo modificado. Se esta correção não for precisa a adesão ficará prejudicada, resultando na baixa resistência da adesão das partículas (Xing et al., 2004). Como foram utilizados os mesmos parâmetros de processo em todos os tratamentos, independente do $\mathrm{pH}$ do adesivo, pode ter havido pré-cura do adesivo formado com $\mathrm{LI}$ ( $\mathrm{pH}$ mais ácido) enfraquecendo a ligação entre as partículas e comprometendo a ligação interna do painel, uma vez que a adesão é decomposta durante a prensagem a quente (Akyüz et al., 2010).

Resultados semelhantes foram observados no estudo de Ferreira (2017) em que somente o tratamento testemunha atendeu aos requisitos das normas. Outros estudos empregando UF modificada como adesivo também encontraram baixos valores, como em Freitas \& Lenz (2019) e Carvalho et al. (2014a) cujos valores de TP variaram entre 0,17 e 0,23MPa, respectivamente. Valores menores que os obtidos no presente estudo. Já Carvalho et al. (2014b) realizaram trabalhos com Pinus caribaea var. bahamensis e tanino de casca de acácia obtendo resultados que variaram entre 0,69 a 1,06MPa, valores muito superiores aos encontrados no presente estudo. Outra propriedade que pode afetar a TP dos painéis é o perfil de densidade. De acordo com Surdi et al. (2014), a TP é em grande parte determinada pela menor densidade que ocorre na camada interna. Adicionalmente, Wong et al. (1999) estudando propriedades de painéis de partículas com diferentes perfis de densidade, concluíram que a TP possui alta correlação com o miolo dos painéis. Como o perfil de densidade (Tabela 4) de todos os tratamentos apresentou grande variação entre as camadas externas e o miolo, pode-se concluir que essa variável também influenciou nos valores de ligação interna. 


\section{CONCLUSÕES}

Apesar das diferenças em suas propriedades físicas e nos processos de extração, ambas as ligninas apresentaram composições químicas semelhantes. Porém, a adição simples das ligninas à UF afetou negativamente todas as características físico-químicas do adesivo, consequentemente, gerou prejuízo nas etapas de cura, quando incorporados aos painéis aglomerados.

A estabilidade dimensional dos painéis foi prejudicada com o acréscimo do percentual de adição das ligninas à UF, sendo que nenhum dos tratamentos atendeu aos parâmetros das normas de referência. Nas propriedades mecânicas observou-se a mesma tendência, onde o aumento gradativo da lignina proporcionou queda dos valores médios de resistência e rigidez.

Como trabalho futuros, sugere-se modificar o processo de mistura das ligninas a resina uréia-formaldeído, por meio de suas razões molares, de forma a tornar o adesivo mais reativo; reduzir a proporção de lignina no adesivo, o que pode facilitar o processo de pulverização, tornando a aplicação do adesivo mais homogênea e reduzindo o tempo na encoladeira; aumentar o tempo de prensagem a quente sob igual temperatura; e testar os painéis quanto a emissão de formaldeído.

\section{AGRADECIMENTOS}

Os autores agradecem as empresas Suzano S.A - Unidade de Limeira (SP), Bonet Madeiras e Papéis Ltda - Unidade de Santa Cecília (SC) e Sudati Painéis Ltda - Unidade de Otacílio Costa (SC); a Universidade do Estado de Santa Catarina - UDESC; a Universidade Federal de Pelotas - UFPel; e a Fundação de Amparo a Pesquisa e Inovação do Estado de Santa Catarina - FAPESC.

\section{REFERÊNCIAS BIBLIOGRÁFICAS}

Akpabio, U. D. (2012). Effect of pH on the properties of urea formaldehyde adhesives. International Journal of Medicinal Chemistry, 2(1), 15-19.

Akyüz, K. C., Nemli, G., Baharoğlu, M., \& Zekoviç, E. (2010). Effects of acidity of the particles and amount of hardener on the physical and mechanical properties of particleboard composite bonded with urea formaldehyde. International Journal of Adhesion and Adhesives, 30(3), 166-169.

American National Standards Institute - ANSI. (2009). ANSI A208.1. Washington: ANSI.

American Society for Testing and Materials - ASTM. (1993). ASTM D1037. West Conshohocken: ASTM.

American Society for Testing and Materials - ASTM. (1998). ASTM D1084-97 (Método B). West Conshohocken: ASTM.

American Society for Testing and Materials - ASTM. (1999). ASTM D2471-99. West Conshohocken: ASTM.

American Society for Testing and Materials - ASTM. (2013). ASTM D1490-01. West Conshohocken: ASTM.

Andrade, L. M. F., Scatolino, M. V., Faria, D. L., César, A. A., Mendes, L. M., \& Guimarães Junior, J. B. (2018). Inclusão do resíduo de polpação da celulose na produção de painéis aglomerados de média densidade. Scientia Forestalis, 46(120), 626-637.

Associação Brasileira de Normas Técnicas - ABNT. (1999). ABNT NBR 14339. Rio de Janeiro: ABNT.

Associação Brasileira de Normas Técnicas - ABNT. (2006). ABNT NBR 14810. Rio de Janeiro: ABNT.

Associação Brasileira de Normas Técnicas - ABNT. (2013). ABNT NBR 14810. Rio de Janeiro: ABNT.

Canadian Standards Association - CSA. (1993). 0437.0-93. Ontario: CSA.

Carvalho, A. G., Lelis, R. C. C., \& Nascimento, A. M. (2014a). Avaliação de adesivos à base de taninos de Pinus caribaea var. bahamensis e de Acacia mearnsii na fabricação de painéis aglomerados. Ciência Florestal, 24(2), 479-489.

Carvalho, A. G., Zanuncio, A. J. V., Mendes, R. F., Mori, F. A., Silva, M. G., \& Mendes, L. M. (2014b). Adesivos tânicos de Stryphnodendron adstringens (Mart.) Coville na produção de painéis aglomerados. Revista Árvore, 38(1), 195-202. 
Chávez-Sifontes, M., \& Domine, M. E. (2013). Lignina, estructura y aplicaciones: métodos de despolimerización para la obtención de derivados aromáticos de interés industrial. Avances en Ciencias e Ingeniería, 4(4), 15-46.

Collares, D. (2015). Aplicações para valorização da lignina. Agroenergia em Revista, 4(9), 62-72.

Comité Européen de Normalisation - CEN. (2003). EN 312-2. Bruxelles: CEN.

Deutsches Institut für Normung - DIN. Normen Für Holzfaserplaten Spanplatten Sperrholz. (1982). DIN 52362. Berlin: DIN.

Eleotério, J. R., Tomazello Filho, M., \& Bortoletto Junior, G. (2000). Propriedades físicas e mecânicas de painéis MDF de diferentes massas específicas e teores de resina. Ciência Florestal, 10(2), 75-90.

El-Mansouri, N. E. (2006). Despolimerización de lignina para su aprovechamiento en adhesivos para producir tableros de partículas (Tese de doutorado). Escola Tècnica Superiora d'Enginyeria Química, Universitat Rovira i Virgili, Tarragona.

Ferreira, J. C. (2017). Sínteses de adesivos de ureia-formaldeído com adição de lignina kraft e celulose nanocristalina (Tese de doutorado). Universidade Federal de Viçosa, Viçosa.

Freitas, A., \& Lenz, D. M. (2019). Produção de painéis de madeira com resíduos de MDF e MDP da indústria moveleira e resina à base de tanino de Acácia Negra. Engevista, 21(1), 141-153.

Gamage, N., \& Setunge, S. (2015). Modelling of vertical density profile of particleboard, manufactured from hardwood sawmill residue. Wood Material Science \& Engineering, 10(2), 157-167.

Giummarella, N., Lindgren, C., Lindström, M. E., \& Henriksson, G. (2016). Lignin prepared by ultrafiltration of black liquor: investigation of solubility, viscosity, and ash content. BioResources, 11(2), 3494-3510.

Iwakiri, S., Shimizu, J., Silva, J. C., Del Menezzi, C. H. S., Puehringher, C. A., Venson, I., \& Larroca, C. (2004). Produção de painéis de madeira aglomerada de Grevillea robusta A. Cunn. ex R. Br. Revista Árvore, 28(6)

Kelly, M. W. (1977). Critical literature review of relationships between processing parameters and physical properties of particleboard (General Technical Report, 66 p.). Madison: U.S. Department of Agriculture, Forest Service, Forest Products Laboratory.

Kim, S., Lee, Y.-K., Kim, H.-J., \& Lee, H. H. (2003). Physico-mechanical properties of particleboards bonded with pine and wattle tannin-based adhesives. Journal of Adhesion Science and Technology, 17(14), 18631875.

Magalhães, M. A., Vital, B. R., Carneiro, A. C. O., Silva, C. M. S., Fialho, L. F., Figueiró, C. G., \& Ferreira, J. C. (2019). Adição de lignina Kraft à resina fenólica para a fabricação de compensados. Ciência da Madeira, 10(2), 142-149.

Maloney, T. M. (1993). Modern particleboard and dry-process fiberboard manufacturing (689 p.). San Francisco: M. Freeman.

Martins, E. H., Guimarães Junior, J. B., Protásio, T. D. P., Mendes, R. F., \& Mendes, L. M. (2014). Painéis aglomerados convencionais produzidos com madeira de Cecropia pachystachya. Enciclopédia Biosfera, 10(19), 1418.

Moslemi, A. A. (1974). Particleboard: materials (Vol. 1, 244 p). London: Southern Illinois University.

Nali, E. C., Ribeiro, L. B. N. M., Hora, A. B. (2016). Biorrefinaria integrada à indústria de celulose no Brasil. BNDES Setorial, 43, 257-294.

Negrão, D. R., Sain, M., Leão, A. L., Sameni, J., Jeng, R., Jesus, J. P. F., \& Monteiro, R. T. R. (2015). Fragmentation of lignin from organosolv black liquor by white rot fungi. BioResources, 10(1), 1553-1573.

Rosa, R. S., Hillig, E. (2014). Utilização de lignosulfonato de amônio na produção de painéis aglomerados. Ciência da Madeira, 5(2), 93-102.

Silva, G. C., Lelis, R. C. C., Oliveira, G. L., Silva, B. C., Lossano, W. C. S., \& Abreu, H. S. (2019). Propriedades de adesivo aplicado em painéis a partir da substituição por lignossulfonato do processo sulfito. Ciência Florestal, 29(1), 322-335.

Spiridon, I. (2018). Biological and pharmaceutical applications of lignin and its derivatives: a mini-review. Cellulose Chemistry and Technology, 52(7-8), 543-550.

Surdi, P. G., Bortoletto, G. J., Castro, V. R., Mendes, R. F., Almeida, N. F., \& Filho, M. T. (2014). Relação entre perfil de densidade e ligação interna de painéis de Pinus spp. Floresta e Ambiente, 21(3), 349-357. 
Technical Association of the Pulp and Paper Industry - TAPPI. (1998). TAPPI T211 om-97. Atlanta: TAPPI.

Trianoski, R., Iwakiri, S., Matos, J. L. M., \& Chies, D. (2013). Utilização da madeira de Cryptomeria japonica para produção de painéis aglomerados. Scientia Forestalis, 41(97), 57-64.

Wong, E.-D., Zhang, M., Wang, Q., \& Kawai, S. (1999). Formation of the density profile and its effects on the properties of particleboard. Wood Science and Technology, 33(4), 327-340.

Wu, Q. (1999). In-plane dimensional stability of oriented strand panel: effect of processing variables. Wood and Fiber Science, 31(1), 28-40.

Xing, C., Zhang, S. Y., \& Deng, J. (2004). Effect of wood acidity and catalyst on UF resin gel time. Holzforschung, $58,408-412$.

You, T., \& Xu, F. (2016). Applications of molecular spectroscopic methods to elucidation of lignin structure. In M. T. Stauffer (Ed.), Applications of molecular spectroscopy to current research in the chemical and biological science (pp. 235-260). New York: InTech.

Zhou, X.-F. (2014). Structural characterization of Kraft lignin for its green utilization. Wood Research, 59(4), 583-592.

Contribuição dos Autores: SST: conceituação, curadoria dos dados, análise formal, investigação, metodologia, administração do projeto, escrita - primeira redação, escrita - revisão e edição; ABC: conceituação, curadoria dos dados, análise formal, investigação, metodologia, administração do projeto, escrita - primeira redação, escrita revisão e edição; PDR: investigação, metodologia, escrita - primeira redação, escrita - revisão e edição; PZ: investigação, metodologia, escrita - primeira redação, escrita - revisão e edição. 